Proceedings of the Online Conference "Applications of Physics in Mechanical and Material Engineering"

\title{
Modeling Droplet Impact on Solids Obstacle of Various Shapes
}

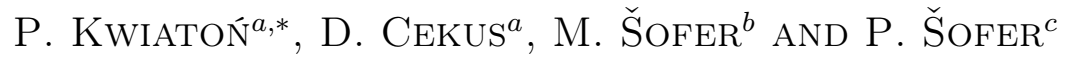 \\ ${ }^{a}$ Department of Mechanics and Machine Design Fundamentals, \\ Faculty of Mechanical Engineering and Computer Science, \\ Częstochowa University of Technology, al. Armii Krajowej 21, 42-201 Częstochowa, Poland \\ ${ }^{b}$ Department of Applied Mechanics, \\ Faculty of Mechanical Engineering, VŠB-Technical University of Ostrava, \\ 17. listopadu 15/2127, 70833 Ostrava-Poruba, Czech Republic \\ ${ }^{c}$ Department of Control Systems and Instrumentation, \\ Faculty of Mechanical Engineering, VŠB-Technical University of Ostrava, \\ 17. listopadu 15/2127, 70833 Ostrava-Poruba, Czech Republic
}

Doi: 10.12693/APhysPolA.139.506

*e-mail: kwiaton@imipkm.pcz.pl

\begin{abstract}
This paper deals with the modeling of the phenomenon of a droplet hitting an obstacle. The numerical model of the multiphase flow is based on the Navier-Stokes differential equations and the finite volume method. During the numerical analysis, the volume of fluid (VoF) method was used as a free surface modeling technique. Various shapes of the analyzed solid obstacles were considered in the numerical model. Two cases of multiphase flows with different surface tensions were analyzed: water-air and gasoil-air. The results were presented in the form of volumetric fraction graphs.

topics: droplet fall, multiphase flow, Euler-Euler approach, VoF model
\end{abstract}

\section{Introduction}

The droplet generation and its impact on the obstacles is an issue that has several applications in industry. Most often, modeling of such a phenomenon concerns the chemical or petrochemical sectors [1]. This process is a complex problem that can be solved using numerical methods based on CFD (computational fluid dynamics).

The phenomenon of droplets hitting an asymmetric surface was modeled in [2]. There, a 3D model was presented in which the surface tension was impelled as a volumetric force acting on the medium in the region of the free surface. An analysis of the dynamics of a water droplet hitting an obstacle made of Kevlar was performed in [3]. Different velocities of the falling droplet were investigated as well as the density of the fluid. The stresses on a flat, Kevlar plate caused by the collision were also determined.

The study of the impact of the surface temperature, impact velocity, and shape on the droplet dynamics was presented in [4]. The influence of normal and tangential velocities as well as the characteristics of the droplet impact regimes on a dry surface was also discussed. The dynamics of a free falling water droplet and its phase changes were described in [5]. Based on the volume of fluid method (VoF), the water-air phase interface was monitored.
An axial-symmetric model was developed in which the changes of fresh and salt water droplets were taken into account.

Apart from numerical studies, one can find many research papers on experimental tests of the droplet modeling phenomenon or its collision with obstacles. The identification and quantification of the characteristics of droplets' impact on metal grids using the VoF method were presented in [6]. Research was carried out using both numerical and experimental methods. The influence of surface tension and liquid viscosity on the adhesive properties of the droplets was determined. Experimental tests of droplet dynamics on a flat surface with the use of microphotography were presented in [7]. Different phases such as water, ethanol, and tetradecane were considered. Particular attention was paid to the influence of surface roughness on the impact dynamics. In [8], the characteristics and regularity of convection processes in the sessile droplet were investigated. Particle movement caused by capillary force and liquid convection was analyzed and the model included surfactant additives.

In this paper, the analysis of the droplet motion dynamics was performed, which included two stages: forming and hitting an obstacle. Four cases differing in the shape of obstacles and the viscosity of the phases were analyzed. 


\section{Methods}

The mathematical model of the droplet dynamics was developed on the basis of the Navier-Stokes differential equations. The mass conservation equation can be written as [9]:

$$
\frac{\partial}{\partial t} \rho_{p}+\nabla \cdot\left(\rho_{p} \boldsymbol{v}_{p}\right)=0
$$

For an infinitesimal moving fluid element, the energy equation takes a form:

$$
\begin{aligned}
& \frac{\partial}{\partial t}\left(\rho_{p} E_{p}\right)+\nabla \cdot\left(\rho_{p} \boldsymbol{v}_{p} E_{p}\right)=-\nabla \cdot\left(\boldsymbol{v}_{p} \boldsymbol{p}_{p}\right) \\
& \quad+\nabla \cdot\left(\boldsymbol{v}_{p} \cdot \boldsymbol{T}_{p}\right)+\boldsymbol{v}_{p} \cdot \boldsymbol{H}_{p}-\nabla \cdot \boldsymbol{H}_{i p}+H_{E p},
\end{aligned}
$$

and the equation of momentum conservation can be written as:

$$
\frac{\partial}{\partial t}\left(\rho_{p} \boldsymbol{v}_{p}\right)+\nabla \cdot\left(\rho_{p} \boldsymbol{v}_{p} \boldsymbol{v}_{p}\right)=-\nabla \boldsymbol{p}_{p}+\nabla \cdot \boldsymbol{T}_{p}+\boldsymbol{F}_{p}
$$

The following symbols are introduced in (1)-(3): $\rho$ - the sharp/dispersed phase density, $\boldsymbol{v}_{p}$ - the flow velocity, $\boldsymbol{p}_{p}$ - the pressure, $\boldsymbol{T}_{p}-$ the deviatoric stress tensor, $E_{p}$ - the total energy, $\boldsymbol{F}_{p}-$ the tensor of external forces, $H_{i p}$ - the heat flux in $p$-phase and $H_{E p}$ - the heat source. A detailed description of the mathematical model of the analyzed problem was included, among others, in [10-15].

The paper presents the process of droplet forming, falling, and hitting against an obstacle. The numerical model (Fig. 1) was developed using the VoF method. The analyzed problem concerns the $2 \mathrm{D}$ case and the two phases: gasoil-air and waterair. The mechanical properties are selected, i.e.,

$$
\begin{aligned}
& \text { - } \operatorname{air}\left(\rho=1.23 \frac{\mathrm{kg}}{\mathrm{m}^{3}}, \mu=1.79 \times 10^{-5} \frac{\mathrm{kg}}{\mathrm{m} \mathrm{s}}\right) \text {, } \\
& \text { - water }\left(\rho=998 \frac{\mathrm{kg}}{\mathrm{m}^{3}}, \mu=1.00 \times 10^{-3} \frac{\mathrm{kg}}{\mathrm{m} \mathrm{s}}\right) \text {, } \\
& \text { - gasoil }\left(\rho=830 \frac{\mathrm{kg}}{\mathrm{m}^{3}}, \mu=3.30 \times 10^{-3} \frac{\mathrm{kg}}{\mathrm{m} \mathrm{s}}\right) \text {. }
\end{aligned}
$$

For the droplet generation process, the user-defined function was implemented, which allowed to determine the appropriate initial inlet velocity. The duration of the numerical tests was $80 \mu \mathrm{s}$. The initial conditions were adopted similar to the study [11]. Surface tension parameters were respectively $0.073 \mathrm{~N} / \mathrm{m}$ for water-air and $0.036 \mathrm{~N} / \mathrm{m}$ for gasoil-air phases. The maximum value of inlet velocity was $11 \mathrm{~m} / \mathrm{s}$.

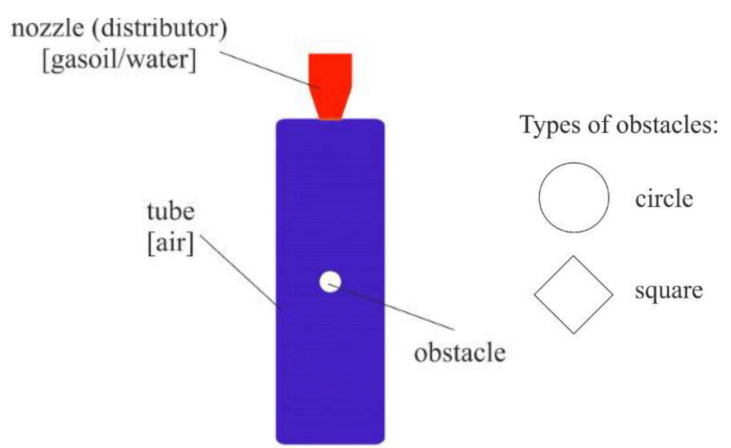

Fig. 1. Model of the analyzed problem.

\section{Sample results}

Numerical tests were carried out for four cases differing in the second phase used and the shape of the obstacle. The influence of the shape of the obstacle on the dispersion of the falling drop was analyzed. Numerical simulations were carried out in the Ansys Fluent software which is based on the finite volume method. The calculations were made on the basis of the NITA (Non-Iterative Time Advancement) solver and Least Square Cell Based for Spatial Discretization. Due to the small dimensions of the analyzed domain, the double-precision option was used.

On the basis of the obtained results of numerical simulations, it can be concluded that the square obstacle (Figs. 2 and 3) causes a much larger irregularity in the droplet decomposition than the circular obstacle (Figs. 4 and 5).

For the circular obstacle, a much larger spread of the liquid in the horizontal direction is visible. For the same time $(t=60 \mu \mathrm{s})$, it was noticed that the water droplet hit the obstacles faster than the gasoil phase. This is related to liquid viscosity and the formation process of the droplet - in the case of the gasoil phase, the process is longer.

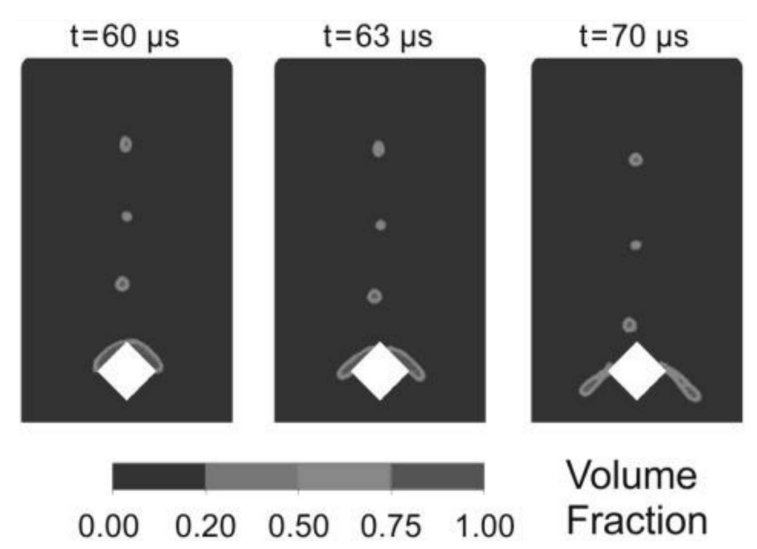

Fig. 2. Volume fraction for water-air phase and square obstacle for a specific time.

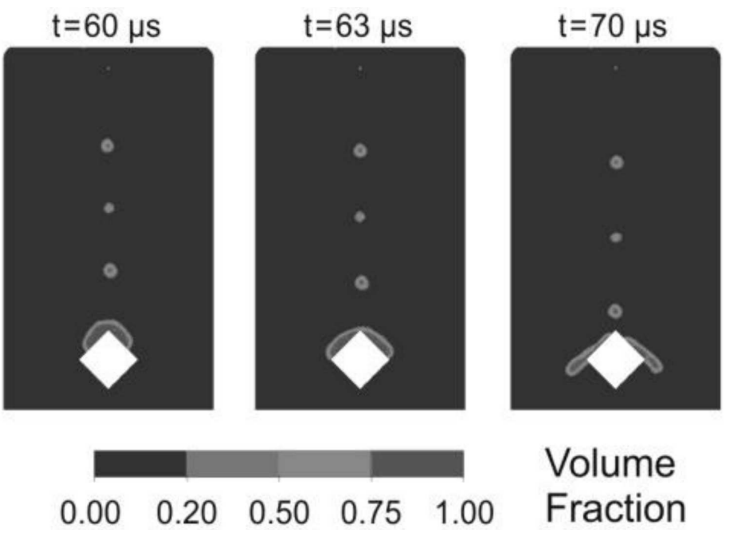

Fig. 3. Volume fraction for gasoil-air phase and square obstacle for a specific time. 


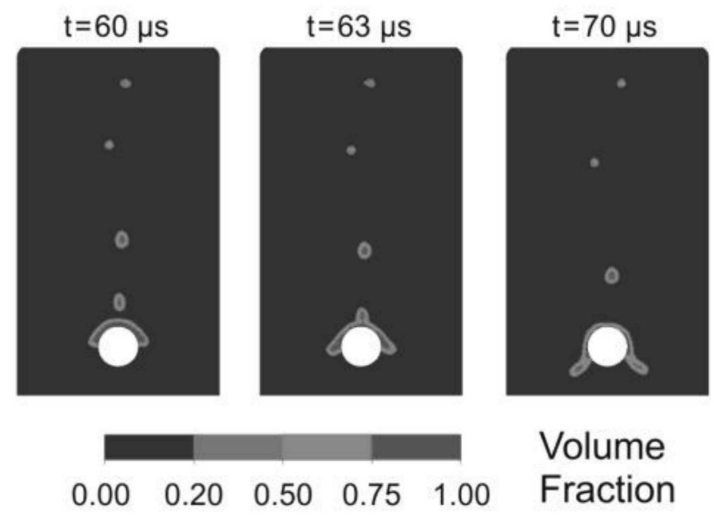

Fig. 4. Volume fraction for water-air phase and circular obstacle for a specific time.

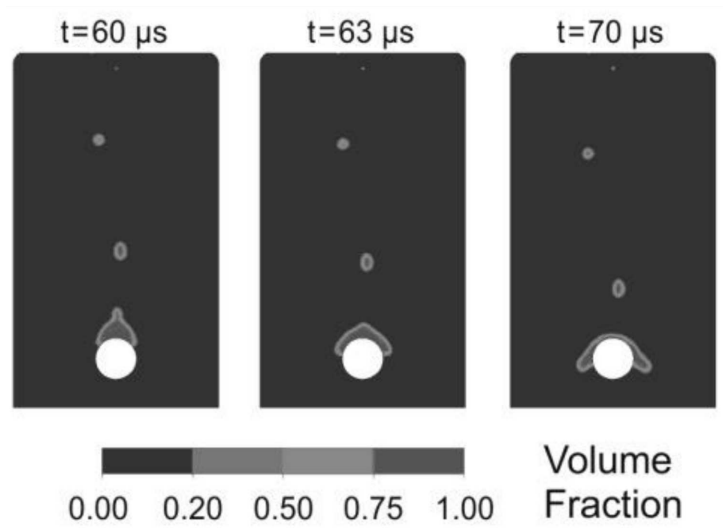

Fig. 5. Volume fraction for gasoil-air phase and circular obstacle for a specific time.

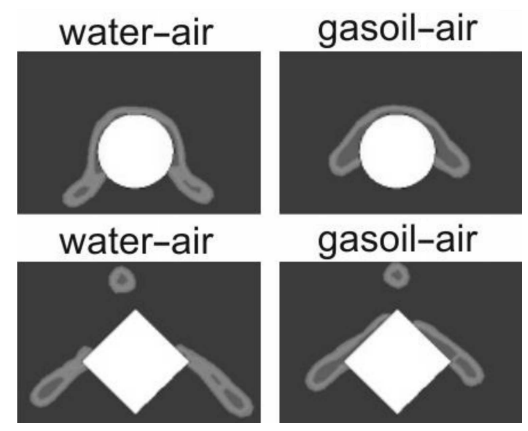

Fig. 6. Comparison of the analyzed cases.

For the square obstacle, a very similar nature of the droplet decomposition was observed for both analyzed liquids. Analyzing the obtained results, one can also notice the formation of micro-droplets of very small diameters during the generation process. For the gasoil-air phases, four such micro-droplets were determined, while for the water-air phases, three of them were noticed. The differences during the impact of the droplet hitting obstacles of various shapes are shown in Fig. 6.

\section{Conclusions}

In this work, the dynamics of a liquid droplet hitting obstacles of various shapes were analyzed. The problem consisted of three stages: formation, free fall, and hitting an obstacle. Two liquids were analyzed as the second phase differing in viscosity value: water and gasoil. The research was carried out in the Ansys Fluent program based on the VoF method and Euler-Euler approach. The analysis was reduced to the $2 \mathrm{D}$ problem. The obstacles were modeled in two ways: as a square and as a circle. This made it possible to determine the influence of the shape of the obstacle on the behavior of the droplet during an impact. It was found that the square obstacle causes a much greater disturbance during droplet separation. There is a characteristic droplet spread in the horizontal direction. In the case of a round obstacle, the droplet flows around it and the spread is much smaller. During the droplet generation process, the phenomenon of several micro-droplets formations was also noticed. The problem presented in this paper will be further developed by changing the inlet velocity and the adhesion parameters of the obstacles.

\section{Acknowledgments}

This work was supported by the European Regional Development Fund in the Research Centre of Advanced Mechatronic Systems project, CZ.02.1.01/0.0/0.0/16_019/0000867 within the Operational Programme Research, Development and Education and the project SP2021/27 Advanced methods and technologies in the field of machine and process control supported by the Ministry of Education, Youth and Sports, Czech Republic.

\section{References}

[1] S.A. Baintabaei, A. Amirfazli, Phys. Fluids 29, 062111 (2017).

[2] M. Bussmann, J. Mostaghimi, S. Chandra, Phys. Fluids 11, 1406 (1999).

[3] S. Nithya, S. Manigandan, J. Devipriya, P. Gunasekar, J. Chem. Pharm. Sci. 974, 2115 (2017).

[4] S. Moghtadernejad, C. Lee, M. Jadidi, Fluids 5, 107 (2020).

[5] K.R. Sultana, K. Pope, L.S. Lam, Y.S. Muzychka, Int. J. Heat Mass Transf. 115B, 461 (2017).

[6] K. Vontas, C. Boscariol, M. Andredaki, A. Georgoulas, C. Crua, J.H. Walther, M. Marengo, Fluids 5, 81 (2020).

[7] C. Tang, M. Qin, X. Weng, X. Zhang, P. Zhang, J. Li, Z. Huang, Int. J. Multiphase Flow 96, 56 (2017). 
[8] S.Y. Misyura, V.S. Morozov, O.A. Gobyzov, Appl. Sci. 10, 4414 (2020).

[9] Z. Saternus, W. Piekarska, M. Kubiak, T. Domański, L. Sowa, Procedia Eng. 136, 95 (2016).

[10] F. Jiang, Y. Wang, J. Xiang, Z. Liu, Chem. Eng. Tech. 38, 1565 (2015).

[11] P. Kwiatoń, D. Cekus, M. Miara, I. KubikMaciagg, Acta Phys. Pol. A 138, 276 (2020).
[12] M. Jiang, B. Zhou, ECS Trans. 83, 127 (2018).

[13] A. Baraldi, M.S. Dodd, A. Ferrante, Comput. Fluids 96, 322 (2014).

[14] B. Silva de Lima, L. de Souza Meira, F. Jose de Souza, Eur. J. Mech. B Fluids 84, 63 (2020). 